\title{
Structural Interventions for HIV Prevention and Care Among US Men Who Have Sex with Men: A Systematic Review of Evidence, Gaps, and Future Priorities
}

\author{
Gregory Phillips II ${ }^{1,2}$ (1) . David McCuskey ${ }^{1,2} \cdot$ Megan M. Ruprecht ${ }^{1,2} \cdot$ Caleb W. Curry $^{1,2,3} \cdot$ Dylan Felt ${ }^{1,2}$
}

Accepted: 16 January 2021 / Published online: 3 February 2021

(c) The Author(s), under exclusive licence to Springer Science+Business Media, LLC part of Springer Nature 2021

\begin{abstract}
The preponderance of HIV interventions have been behavioral, targeting individual, dyadic, or group dynamics. However, structural-level interventions are required to decrease HIV transmission and increase engagement in care, especially for men who have sex with men (MSM), particularly Black and Latinx MSM. A systematic literature review was conducted to assess the current state of structural interventions; only two studies detailing structural interventions related to HIV for Black and Latinx MSM in the US were identified. An additional 91 studies which discussed structural-level barriers to optimal HIV outcomes among MSM, yet which did not directly evaluate a structural intervention, were also identified. While this paucity of findings was discouraging, it was not unexpected. Results of the systematic review were used to inform guidelines for the implementation and evaluation of structural interventions to address HIV among MSM in the U.S. These include deploying specific interventions for multiply marginalized individuals, prioritizing the deconstruction of structural stigma, and expanding the capacity of researchers to evaluate "natural" policy-level structural interventions through a standardization of methods for rapid evaluative response, and through universal application of sex, sexual orientation, and gender identity demographic measures.
\end{abstract}

Keywords Structural interventions $\cdot$ HIV $\cdot$ Prevention $\cdot$ Men who have sex with men $\cdot$ United States

\section{Introduction}

In 2018, an estimated 37,800 people in the United States (US) were diagnosed with HIV. Nearly $70 \%$ of all new infections in 2018 were among gay, bisexual, and other men who have sex with men (MSM), with marked disparities among racial/ethnic minorities-Black MSM (BMSM) and Latinx MSM (LMSM) accounted for 67\% of HIV diagnoses among MSM, but comprise only $11.6 \%$ of the MSM population in the US $[1,2]$. Despite the perception that disparities are

Gregory Phillips II

glp2@northwestern.edu

1 Evaluation, Data Integration, and Technical Assistance (EDIT) Program, Institute for Sexual and Gender Minority Health and Wellbeing, Northwestern University, $625 \mathrm{~N}$ Michigan Ave \#14-043, Chicago, IL 60611, USA

2 Department of Medical Social Sciences, Northwestern University Feinberg School of Medicine, Chicago, IL, USA

3 Case Western Reserve University Undergraduate Studies, Cleveland, OH, USA driven by behavioral factors such as condomless sex and multiple sex partners, the reality is that a complex array of multilevel factors beyond just individual-level behaviors has led to the disproportionate rates of HIV among MSM, particularly racial/ethnic minority MSM [3]. Among MSM, it is now known that: poverty leads to a lack of access to health care and preventive services [4]; instability in housing results in an inability to maintain viral suppression or take pre-exposure prophylaxis (PrEP) regularly [5]. Further, racism/homophobia/biphobia results in a lack of culturally responsive care and subpar service provision in healthcare settings [6, 7], is linked to the creation of hostile environments in other major social systems, including housing, education, and criminal justice [8-11], and has been further associated with HIV risk behaviors [12]. Mass incarceration impacts both infection rates and engagement in care [13]. Moreover, these barriers do not operate in isolation: individual-, interpersonal-, community-, and structural-level factors combine to create extensive, multilevel barriers to HIV prevention and care among sexual, gender, and racial/ ethnic minority populations $[4,14-16]$. 
Given the multilevel drivers of HIV infection, particularly among minoritized communities, there is an obvious need to continue expanding the research literature on intervention methods beyond the individual level. However, most funded HIV prevention and treatment interventions are behavioral in nature, and focus largely on individual, dyadic, or group dynamics. According to a recent search of NIH RePORTER, only 337 of 1701 funded HIV interventions (19.8\%) contained the key word "structural," and the vast majority of these interventions were not specifically focused on changing an aspect of the structural environment [17]. Although behavioral interventions often play a valuable role in improving the livelihoods of individuals, and can have real and tangible effects on HIV attitudes, beliefs, and safer sex practices, they are limited in their ability to effect change at the population level. For one, behavioral interventions often require substantial resources for minimal benefit at scale [18]. Additionally, they frequently fail to address the social contexts in which behavior-and therefore, elements of health—occur, and face a wide range of challenges related to larger, ideological macro-systems (racism, sexism, homophobia, biphobia, etc.), which play out in other structural and material realities, such as infrastructure, network- and sociallevel contextual factors, and population-level distributions of capital investment [18-22]. Moreover, many critiques have been made about the limited long-term efficacy of behavioral interventions, the strength of which is known to fade over time, resulting in only a short-term, temporary adjustment to behavior [23, 24]. In addition to these practical shortcomings, there have also been a number of vital philosophical critiques levied at behavioral-only approaches to HIV elimination which fail to account for structural concerns, including their tendency to conceptualize the individual—and specifically, often marginalized individuals - as the locus of HIV risk, as opposed to the broader systems and structures which act upon individuals; a consistent shortcoming of the field of public health overall [25].

These limitations of behavioral interventions present the need for more comprehensive approaches to HIV from both an academic and program implementation perspective. As such, it is important that we examine our progress in enacting structural change as a field. Public health professionals have long called for a more comprehensive, equity-based approach to the epidemic - one that will address the larger systemic forces driving HIV disparities [26-28]. Among these calls is a consistent theme of advocacy for the implementation, evaluation, and scale-up of structural interventions. Blankenship, Friedman, and colleagues were some of the first to examine structural interventions for HIV in the dominant academic literature; their 2000 systematic review was one of the first to comprehensively examine and typologize approaches to structural interventions. In this review, they came to define structural interventions as "interventions that work by altering the context within which health is produced or reproduced" [29]. We use this same definition in this manuscript.

From such early discussions, socioecological frameworks for HIV prevention have emerged, such as Mugavero's framework for factors influencing engagement in HIV medical care [30] and Meyer's minority stress framework [31]. Singer's exploration of biosocial disease interaction echoes such approaches [32]. Prado, Lightfoot, and Brown called for the creation, evaluation, and dissemination of "interventions that target macro-level factors" in prevention among ethnic minority youth [33]. This work has contributed to and driven a robust body of theory, academic discourse, and advocacy for structural interventions. Ultimately, this body of scholarship coalesces around the need for interventions that aim to influence factors at the macro-system level-that is, "the broad social and philosophical ideals that define a particular culture"-which often involves targeting issues such as poverty, stigma, discrimination, racism, sexism, and homophobia, commonly referred to as "root-causes" of disparities, or as social determinants of health, that contribute to HIV acquisition risk and poor access to HIV services $[3,33,34]$, among other suboptimal outcomes. Structural interventions seek to ameliorate the effects of social determinants of health by counteracting the systemic mechanisms that link these determinants to poorer health outcomes. This could include implementing new policies to improve access to housing and employment, providing additional opportunities for social and economic development for individuals and communities, or programs which sustainably increase availability and accessibility of otherwise inaccessible prevention tools like PrEP or condoms. In other words, the difference could be summarized by stating that behavioral interventions seek to change the way people act, while structural interventions seek to change the environment in which they act [35].

Due to the time, cost, and political will needed, there have not been many large-scale structural interventions enacted in the United States, despite frequent discussion of their potential impact over the last two decades in the HIV/AIDS literature [29, 36]. However, this growing body of work points to the utility of accounting for structural factors in the study of HIV epidemiology. In other words, whether or not one is proposing to actually change structural factors, their role in any given dynamic of HIV prevention or treatment should be explicitly considered. For example, exploring how these factors operate at the community level is useful in examining the pathways between macro-level factors and on-the-ground health outcomes. This could include, among other things, study of the role of racial and economic geospatial segregation in HIV transmission dynamics; a factor which has gained recognition as a key driver of HIV outcome disparities [22, 37-39]. Here, applying a structural lens has allowed researchers to better understand how concentrated pockets of 
HIV vulnerabilities such as poverty status, employment, and homelessness highlight the socioeconomic distribution of health inequality $[40,41]$. Moreover, this framing allows us to see how the distribution of socioeconomic capital makes barriers to care such as cost and health insurance coverage even more pertinent in communities [4, 42, 43]. Similarly, a number of studies have found links between neighborhood socioeconomic context, resource availability, care engagement, and viral suppression [44, 45]. For example, historically Black and resource-deprived neighborhoods are less likely to have an HIV care clinic nearby, therefore increasing travel time to appointments and reducing retention in care [46].

As the example of neighborhood context makes clear, social and socioeconomic factors-including but not limited to homelessness/unstable housing, employment, incarceration, neighborhood effects, health system access, stigmainduced violence, racial/ethnic discrimination, and undocumented status - are key in understanding the larger scope of structural barriers and have been shown to produce inequity in HIV outcomes particularly in racial/ethnic minority MSM populations [4, 42, 47-49]. At the center of these structures and the ways in which they act upon racial/ethnic minority MSM is the systemic production of racism, xenophobia, homophobia, and biphobia, among other forms of stigma, discrimination, and prejudice. These driving forces are apparent across all of these key structural factors including but not limited to the neighborhood effects described above. These patterns have been frequently characterized by researchers as evidence of the role of stigma and discrimination as fundamental causes of inequity [50, 51]. For example, incarceration rates among BMSM reflect the inherent racism, nationalism, and homo/biphobia of the U.S. criminal justice system itself, which is then actualized, among other ways, in the criminal justice system's role in producing disparities in HIV [52-54]. Similarly, housing instability and housing discrimination directly attributable to racism, homo/ biphobia, and other forms of discrimination are a common experience among BMSM and LMSM [55]; as this discrimination produces injustices in housing, so then do injustices in housing produce subsequent inequities in HIV outcomes [56].

In sum, the idea that fundamental, structural factors are driving disease inequity is not novel, and remains a vital area of HIV and public health scholarship. However, despite these rich discussions surrounding the need for structural interventions in HIV research, the persistent and known disparities in HIV acquisition, and an understanding of a number of driving structural factors, the field has largely yet to bring such interventions to fruition. With this research history in mind, we conducted a systematic literature review on HIV structural interventions for Black and Latinx MSM in the US. We discuss results within the context of previous, behaviorally-based HIV interventions and their inability to challenge the systems of power and structural status quo that has contributed to the cyclical harms visited on those most vulnerable to HIV, and emphasize the need for innovative structural interventions to equitably address the HIV epidemic. Despite a paucity of results, we present the resulting manuscripts and outline strategies for researchers and evaluators to implement and evaluate future structural interventions to create a more equitable plan for ending the HIV epidemic.

\section{Methods}

\section{Protocol and Registration}

In order to assess the current status of structural interventions in the US among Black and Latinx MSM, we conducted a two-tiered systematic literature review. This study was designed and reported according to the Preferred Reporting Items for Systematic Reviews and Meta-Analysis (PRISMA) tool [57]. No review protocol has been published for this study, though all analytic methods and inclusion criteria were specified and documented in advance.

\section{Eligibility Criteria}

Studies were considered eligible if they were published in a peer-reviewed journal, took place in the US, focused on HIV, included a primary study population of MSM, depicted a structural intervention (i.e., worked by altering the context within which health is produced or reproduced) [29], and were written in English (due to the linguistic limitations of the authorship team). Papers were included if they specifically discussed LMSM or BMSM; they did not need to be solely focused on these populations to be included. Studies which met all other criteria and discussed structural factors but did not depict a structural intervention were analyzed separately.

\section{Information Sources and Search}

All searches were conducted in October 2019, and all manuscripts published through October 2019 were included. Databases searched include: PubMed, Google Scholar, Web of Science, SCOPUS, Global Health, CINAHL, MEDLINE, ECONLit, PsycINFO. The following search terms were used: (HIV or AIDS) AND (MSM OR "men who have sex with men" OR (men AND (gay OR bisexual OR "sexual minority”))) AND (US OR “United States") AND (structural OR "structural intervention" AND intervention OR policy) AND (black OR latino OR latinx OR hispanic OR “African 
American" OR "African-American" OR "racial minority" OR "ethnic minority").

\section{Study Selection}

Titles were screened and duplicates were removed. Articles were also removed if they were not published in peerreviewed journals, if they were not in English, or if the full text was not available. Then, the study team screened all abstracts to determine if the intervention took place in the US, was focused on HIV, was focused on MSM, discussed LMSM or BMSM, and whether it depicted a structural intervention. Two members of the study team independently screened abstracts for these criteria with a third member providing arbitration on all disputes. Abstracts that met all criteria then proceeded to full article review.

Each article selected for full review was read in full by a group of four researchers who discussed the articles, then voted on which articles met the criteria for inclusion (see Eligibility Criteria). A parallel process took place to determine inclusion of articles which discussed structural factors but did not depict a structural intervention. Majority rule was used to decide in cases of disagreements. There was a plan for tie breaking in place, though this was not needed.

\section{Data Collection and Abstraction}

The resulting articles, both for structural interventions and for structural factors, were analyzed and compared for common themes. Studies were assessed for the following characteristics: structure intervened upon, impact, study population (e.g., age, race/ethnicity), methodology, primary outcome(s), duration of intervention, location, and study setting.

\section{Results}

Having extensive familiarity with the literature prior to this review, the authors were aware of the scarcity of structurallevel HIV interventions for MSM, particularly Black and Latinx MSM. With this in mind, articles that met the initial criteria (US-based, HIV-focused, and involving MSM, including Black and Latinx MSM) were examined in two separate categories: those that examined structural-level factors but did not seek directly to change them, and those that implemented and/or evaluated interventions at the structural level.

\section{Structural Interventions}

The search process resulted in 710 initial results from journal databases (Fig. 1). After removing duplicates, 387 titles and abstracts were screened for inclusion. Of these, 8 manuscripts met all structural intervention eligibility criteria, and their full-text documents were reviewed for verification. While many interventions, such as linkage to care or prison re-entry programs dealt with structural factors, they did not meaningfully change the larger structures in which individuals operate. Rather, they aided those involved in navigating existing systems; we therefore did not consider them structural interventions as defined for the purposes of this systematic review, and they were excluded. During the full-text screen, studies were excluded based on author discussion and determination that the interventions themselves were not implemented at the structural level $(n=6)$. Ultimately, only 2 articles matched all criteria and were deemed to be structural interventions by the authors, indicating a widespread lack of HIV-focused structural interventions for Black and Latinx MSM in the US.

The two interventions included are the Louisiana Public Health Institute's AIDS Initiative for Minority Men (AIMM) and the Adolescent Trials Network for HIV/

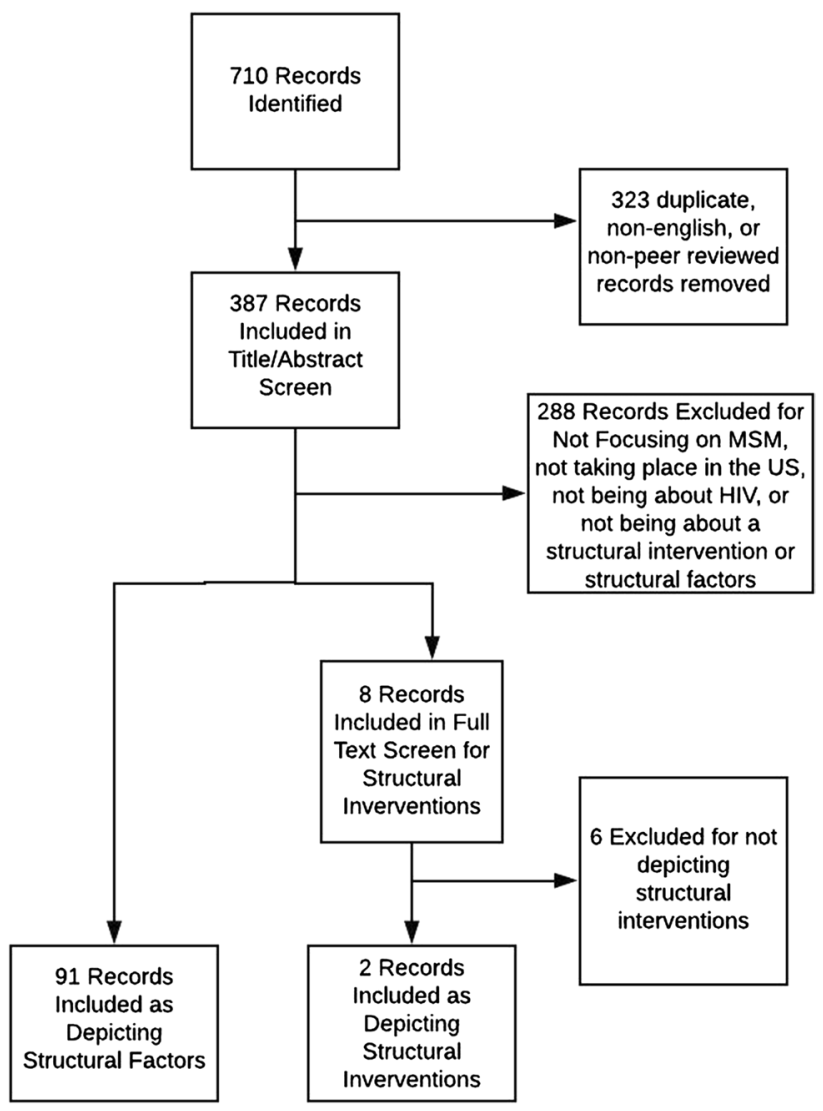

Fig. 1 Record screening process 
AIDS Interventions' Connect to Protect (C2P) project [58, 59]. Both interventions included elements of multilevel approaches to HIV prevention such as testing, building self-efficacy, treatment adherence, and service connectivity. AIMM focused on multilevel, interdisciplinary direct service approaches for a specific population (i.e., BMSM), while $\mathrm{C} 2 \mathrm{P}$ focused on high-level policy and organizational processes across a more diverse range of populations. AIMM took an interdisciplinary direct services approach for BMSM in two different cities [58], contrasting with the high-level policy and organizational processes approach for a wider range of populations affected by HIV across a larger geographic region using nationwide coalitions of C2P [59]. The programs of AIMM were, as such, all specifically targeted towards HIV prevention and treatment needs of BMSM. Leveraging community health centers, community-based service organizations, relationships with historically Black colleges and universities (HBCUs), community centers, as well as community advisory boards (CABs), AIMM implemented programming involving health and social service navigation, case management, education, job coordination, housing outcomes, stigma reduction, and safe space creation [58]. C2P, in contrast, used a wider, coalition-based approach. Coalitions consisted of localized adolescent medicine trial units implementing various population-specific initiatives aimed at lowering adolescent HIV prevalence, with a majority focusing on young MSM (YMSM) [59].

While AIMM had clearly defined city boundaries within Louisiana and a singular target population of BMSM, coalitions for $\mathrm{C} 2 \mathrm{P}$ were established across the US in areas with concentrated prevalence of HIV. Each coalition, comprised of youth from the surrounding community area, targeted local policies, institutional practices and relationships, environmental conditions, resource availability, with an overarching focus on issues related to individual coalitionspecific needs [59]. Examples of structural-level objectives implemented by $\mathrm{C} 2 \mathrm{P}$ include amendments to HIV testing permission laws, linkages between juvenile detention centers and HIV testing, revised testing referral policies, and sexual diversity training programs for public health officials. This approach allowed for more leniency in structural partnerships, contrasting with AIMM's more clearly defined integrated care partnerships consisting of primary care providers, behavioral health providers, AIDS service organizations, and community-based organizations [58].

Results appear to show support for the use of populationspecific approaches to HIV prevention. AIMM showed that the majority $(62 \%)$ of participants living with HIV were linked to care and $49 \%$ maintained viral suppression. Successes were demonstrated in a number of other realms: over $40 \%$ of participants gained employment throughout the intervention, and housing discrimination was identified in 37\% of mystery shopper tests implemented. 15 cases of syphilis were identified at local HBCU events, and a safe space at a community organization was successfully established [58]. $\mathrm{C} 2 \mathrm{P}$ appeared to have more constraints. Identifying overarching goals and outcomes was difficult considering interventions and outcome targets were coalition specific. Thus, the evaluation examined broader factors such as satisfaction with community support, perceived meeting of needs, and internalized HIV stigma. It found that, while youth participants from non-YMSM populations reported greater satisfaction with their community's support and that their needs were better met by community resources relative to year 1, YMSM did not experience such changes. In addition, internalized HIV stigma increased over time for non-YMSM communities, and remained unchanged amongst YMSM, with varying results suggesting that it may be "unreasonable to assume identical chains of structural causality across youth populations who have such different historical relationships to HIV and who encounter very different kinds of entrenched discrimination within their communities" [59]. While there were evidently evaluation constraints to examining overarching intervention efficacy across widely variant, coalition-specific interventions themselves, AIMM shows promise in that it demonstrated multilevel structural effects. The intervention resulted in service connectivity in target communities, improved health and economic outcomes for individual participants, and established additional community infrastructure.

\section{Structural-Level Factors}

During the systematic review process, we uncovered a substantial number of records $(n=91)$ which reported on structural-level factors as primary outcomes, though they lacked a structural-level intervention itself. As such, while these studies were excluded from the primary review, a secondary, parallel review was undertaken, which included analyses of their foci, study population, outcomes, and methodologies (Supplementary Table 1).

Each included study addressed at least one structurallevel factor relating to HIV transmission, prevention, care, or a combination of factors. Figure 2 shows the distribution of structural-level factors within these articles. Stigma (including homophobia and racism) was the most frequently reported factor and was examined in nearly half (49\%) of the 91 articles, followed by cost/insurance/access-related barriers to care (33\%), social/community support (29\%), and overall socioeconomic status (23\%). Other pertinent structural elements appeared less frequently; these included housing (12\%), culturally-competent services (19\%), employment (12\%), neighborhood (11\%), and organizational connectivity and management (16\%). Factors pertaining to the most marginalized populations were largely unexamined: incarceration (10\%), immigration status (7\%), medical mistrust (3\%), 


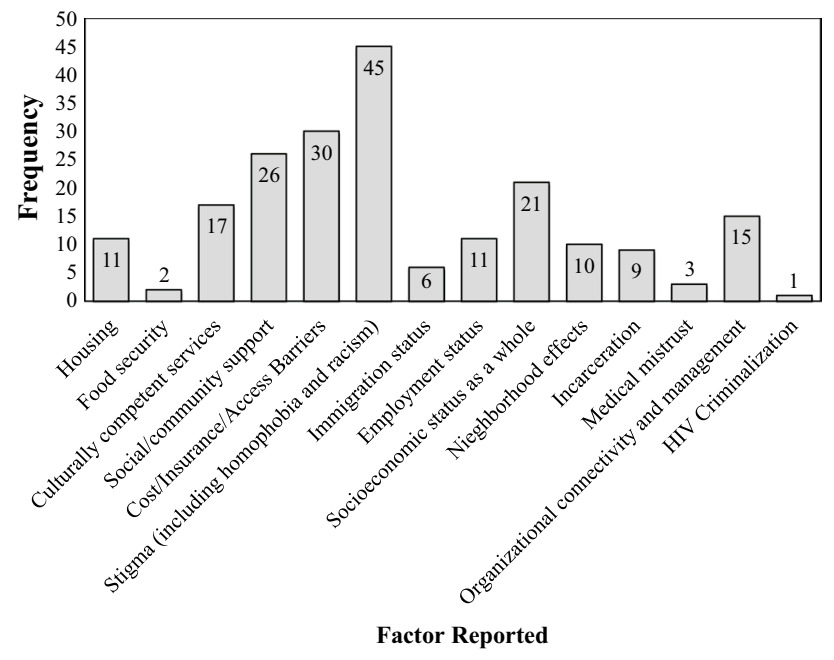

Fig. 2 Structural level factors examined in excluded records

food insecurity (2\%), and HIV criminalization laws (1\%) were the least commonly analyzed structural factors out of studies included in this review.

The included studies on structural factors were further analyzed based on specific study population (Fig. 3). Though all studies included BMSM and LMSM in some way, $42 \%$ of articles did not have a specific racial/ethnic population of focus $(n=38)$. However, $35 \%$ focused on BMSM $(n=32)$, $13 \%$ on LMSM $(n=12)$, and $10 \%$ on BMSM and LMSM studied together $(n=9)$. In regards to age of study population, roughly one-quarter $(n=23)$ of articles specifically focused on YMSM.

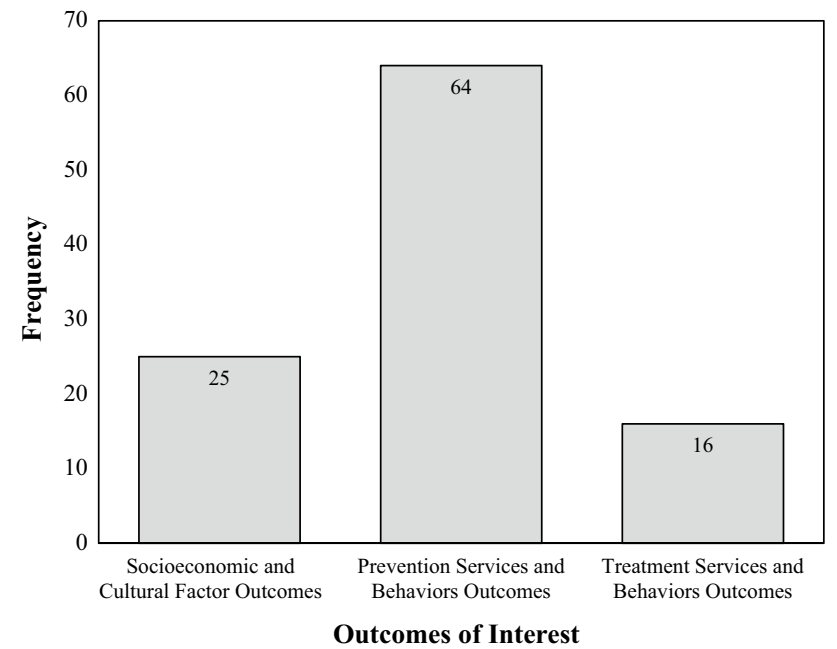

Fig. 3 Distribution of Outcomes of Interest for Studies which examined structural factors
Although outcomes of interest in the 91 articles varied widely, they could be broadly placed into three categories (Fig. 3):

1. Prevention and behavioral change: The greatest number of studies $(70 \%, \mathrm{n}=64)$ focused on outcomes pertaining to prevention services and behaviors, including reducing HIV risk behaviors, prevention service utilization (PrEP access, HIV testing), resiliency, and selfefficacy.

2. Socioeconomic and cultural factors relating to HIV risk and treatment: Fewer $(27 \%, \mathrm{n}=25)$ studies prioritized socioeconomic and cultural factors that enable treatment and prevention, including housing, employment, health care access, and cultural responsiveness of providers.

3. HIV continuum of care and mortality: Only $18 \%$ of studies $(n=16)$ focused on treatment service and HIV care including retention in care and treatment outcomes.

Some studies examined more than one outcome; thus, the overall $\mathrm{n}$ is higher than the number of structural factor studies included.

In terms of methodology, included studies varied widely (Fig. 4). Approximately one-third $(38.5 \% ; n=35)$ of studies were cross-sectional, $25.3 \%(\mathrm{n}=23)$ were qualitative analyses (i.e., focus groups, interviews, ethnographies), $16.5 \%$ $(n=15)$ were reviews or meta-analyses, and $12.1 \%(n=11)$ were cohort studies. The remainder consisted of 4 evaluations, one mixed methods study, one conceptual framework, and one symposium commentary.

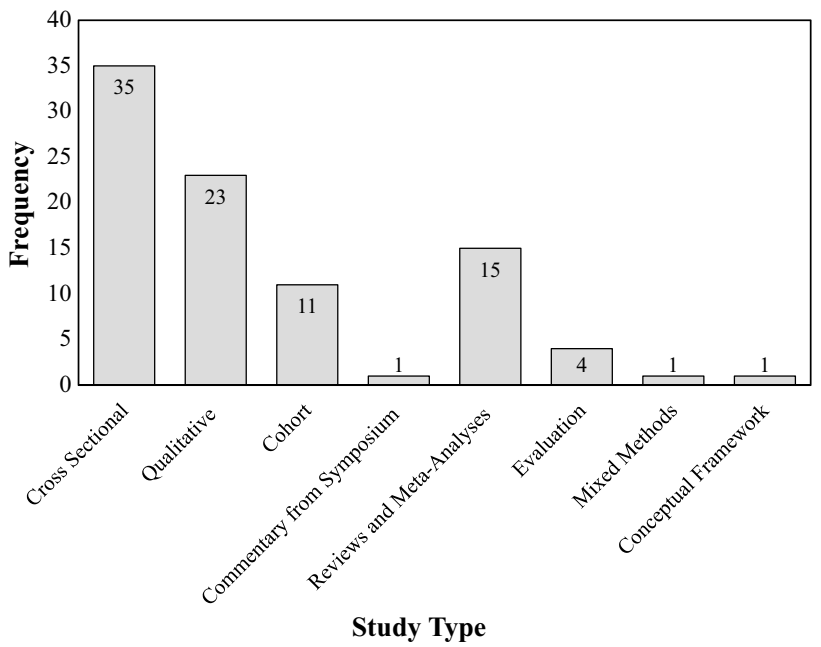

Fig. 4 Frequency of Study Type of Structural Factor Articles 


\section{Discussion}

The results of our systematic literature review are discouraging, but not surprising. As became abundantly clear over the course of our review, there is a serious dearth of literature available on the topic of structural interventions for HIV prevention and care among Black and Latinx MSM in the US, despite an evident need. This gap also points to funding deficits and a lack of available prior research that may make developing and implementing structural interventions to address HIV in this population more difficult. However, these results also represent a vital opportunity to stimulate novel research and structural interventions in this area and illuminate target pathways for potentially impactful future work. Below, we lay out several considerations to guide this work, with a focus on the role of the researcher in advancing structural intervention work.

\section{Priority Populations}

Clearly, MSM, especially Black and Latinx MSM, must be a priority target for future structural interventions, given both their known vulnerability to HIV and the paucity of literature available on structural interventions designed to improve the health of this population. Marginalized individuals who experience unique and intersecting forms of oppression should be given distinct focus and priority in structural intervention research and implementation. For example, BMSM are known to experience distinct forms of enacted and structural stigma as a result of intersecting forms of stigma and discrimination, including homo/ biphobia, racism, and HIV-related discrimination [39]. This results in challenges in accessing HIV prevention and care services $[43,60,61]$ and a disproportionate HIV prevalence $[62,63]$. Though many structural change efforts may have broader impacts which could improve HIV outcomes as a whole (such as increasing the affordability and accessibility of health insurance), it is still vital to ensure that structural change efforts are tailored to meet the distinct needs of these subpopulations. For example, LMSM may be more impacted by an intervention which decreases barriers for undocumented individuals in accessing health services. Structural interventions such as reparations or which aim to improve trust in the medical system may be more important for BMSM specifically.

It should also be noted that Black and Latinx MSM are not the only populations that experience structurallevel barriers to HIV prevention and care. In particular, we encourage additional focused study of existing structural barriers and interventions to HIV prevention and care among transgender individuals, non-binary people who may or may not identify as transgender, Black and Latinx individuals more generally_including Black and Latinx cisgender women, undocumented residents of the US, and sex workers. Each of these demographics faces unique barriers to HIV prevention and care [4, 42, 43, 64-71] and each experiences disproportionate HIV incidence and prevalence $[63,66,70,72-76]$.

\section{Structural Stigma as a Focus of Future Structural Interventions}

By a wide margin, the greatest number of papers which addressed structural barriers to HIV-related health among Black and Latinx MSM focused on either enacted or structural forms of stigma as key points in ensuring equitable outcomes (Fig. 2). Moreover, other structural barriers we observed as important within the literature, such as medical mistrust, culturally-responsive services, and HIV criminalization, are tied to enacted and structural stigma, indicating that the vast majority of manuscripts we identified focused on stigma. While this does not necessarily imply that structural stigma is the most significant or important structural barrier-merely that it is among the most researched and published on-the importance of stigma in structural interventions is highlighted by the mixed results observed in the structural intervention described by Miller et al., wherein YMSM received less community support than heterosexual peers [59]. Moreover, the successes of the intervention reported by Brewer et al., which included stigma reduction as a key component, provide further evidence of the importance of addressing stigma as a priority in structural change efforts [58].

Based on these findings, interventions that target and seek to ameliorate or eliminate structural stigma have the potential to lead to significant improvement in HIV outcomes among Black and Latinx MSM in the US. As well as recommending the prioritization of funding for structural interventions to address disproportionate HIV rates among MSM, we also strongly encourage funders, including the Centers for Disease Control and Prevention (CDC) and the National Institutes of Health (NIH), to include a focus on reducing and/or eliminating structural stigma within their research and practice portfolio, including putting out specific funding opportunities for structural level interventions. Critically, some institutes within NIH have already taken such measures, including the National Institute of Mental Health, National Institute on Alcohol Abuse and Alcoholism, and National Institute on Minority Health and Health Disparities [77-79]. Additionally, it will be important for funders themselves to apply a critical equity lens to their own funding portfolios. Structural interventions are difficult to measure and fund-standardized metrics across populations and institutions are challenging to develop, particularly 
on strict timelines, and research topics such as community interventions and health disparities receive less competitive scores from reviewers [80]. Of vital note-Black applicants are more likely to propose such approaches to the NIH and, also more likely be rejected for funding than their white counterparts [81]. This pattern indicates a need to consider grant funding as a site for structural intervention within the academy to promote and support necessary structural change efforts.

Furthermore, stigma remains a multidimensional concept: in future research, specific drivers and dimensions of stigma, including enacted, structural, and internalized forms, should be continually measured in order to inform the development of more effective interventions related to stigma. In addition to stigma as a broader category of focus, the AIMM and $\mathrm{C} 2 \mathrm{P}$ interventions highlighted the value of community safe spaces and broader community engagement as catalysts for organizing to advance structural change [58, 59]. Moreover, safe spaces such as those established within the AIMM intervention could potentially serve to ameliorate the impacts of stigma as a barrier to structural change in the contexts of community organization and HIV prevention [82]. Given the broad array of potential targets for structural interventions (Fig. 2), we recommend the incorporation of safe spaces and community organizing, as well as targeted efforts to address or account for stigma regardless of whether or not stigma is the primary intervention target, into any proposed structural intervention.

\section{Recommendations for Structural Intervention Development and Implementation}

Although at present there is a highly limited sample of studies, early evidence indicates that targeted, populationspecific structural interventions with clear geographic or community boundaries may be better positioned for success. Significant additional work needs to occur before this hypothesis can be meaningfully tested. Notably, both studies selected featured community-engaged approaches, which is an obvious strength. However, the broader definition of community within the $\mathrm{C} 2 \mathrm{P}$ study created a dynamic wherein community members who were not members of the most affected population (YMSM) reported greater satisfaction with the results of the intervention than YMSM themselves [59]. This again supports the value of establishing protocol boundaries to allow for increased focus on and engagement with target populations.

Prior work in fields such as social-ecological theory has described the function of "leverage" points in systems change- that is, target areas within broad, complex systems where small change can ripple out to produce much larger effects [83]. In contrasting the two studies included in this review, it is apparent that AIMM selected specific leverage points within a local, contained health system-which may have supported success-as compared to the broader, more flexibly defined model contained in the $\mathrm{C} 2 \mathrm{P}$ study. This greater focus, as discussed above, is also potentially attributable to having established clearer boundaries regarding population and geography.

Given our prior recommendations of priority populations and areas of focus, structural interventions which seek to address the impact of stigma on subpopulations of sexual and gender minority (SGM) groups such as MSM (particularly BMSM, LMSM, and YMSM) should ensure that the interventions' boundaries are clearly delineated and target leverage points are established prior to implementation. These recommendations, while again based on small sample sizes, are consistent with prior recommendations made in the literature which have highlighted the importance of contextually-responsive design and authentic community engagement to structural intervention success [36]. Additionally, to ensure success, allies and accomplices, including evaluators, policy analysts, and researchers, who may have greater access to resources or formal training in structural intervention design, are also needed to serve as champions and supporters for community-led structural interventions. Those seeking to take on an allyship or accomplice role in this work should reflectively and critically consider how they can best act to disrupt unjust systems and support the structural changes necessary to equitably address HIV.

\section{Novel Approaches to Structural Intervention Evaluation}

One may rightly assume that, surely, there have been more than two examples of structural changes which had relevant impacts on the HIV-related health of US Black and Latinx MSM. We would agree with this hypothetical claim, and therefore posit that a key missing piece of the puzzle is the ability to measure the impacts of structural change which derives from outside of the academy. In other words, changes brought about through social movements or legislative change may be more difficult to measure and therefore less likely to be captured within the academic literature. Thus, the establishment of new systems to support the evaluation of structural change and its impact on key health indicators are needed. Given that structural interventions could encompass actions such as changes in law, policy, or federal guidance (what could be considered "natural" structural changes as opposed to research-manufactured changes), there can be numerous challenges to evaluating their effect. When structural interventions are developed, implemented, and evaluated by researchers, the necessary infrastructure is in place to methodically study their impact on target outcomes. Of course, the same structure is not available in the case of the passage of a new law with implications for population 
health. This means that many structural changes which may very well have impacted the HIV-related health of Black and Latinx MSM have not had their precise impact in this regard evaluated. For example, as a result of the passage of the Affordable Care Act (ACA) some states made the choice to expand Medicaid, resulting in increased health insurance coverage for sexual minority Americans [84], which could very likely have had significant impacts on how MSM, especially Black and Latinx MSM, engaged in HIV prevention and care. However, no work of which we are aware has evaluated whether or not the ACA impacted relevant HIV health indicators among this population. Moreover, it should be noted that the impact of structural change will not necessarily be felt on the same timeline as individual behavioral change-in fact, it may take months, or even years, before the effect of a change in structural policy can be appropriately studied, or inferences made.

In light of these challenges, we propose two potential actions to facilitate evaluation of structural policy change, prioritizing high-quality evidence in both the short- and long-term. The first of these is the creation of standardized methods for rapid-response approaches to assessing the short-term public health consequences of structural change. Again, take the example of US health insurance law. Any changes to US insurance policy should be evaluated for their actual impact on access to care and short-, medium-, and long-term health outcomes. For example, a recent publication in The Lancet concluded that repeal of the ACA would worsen overall health outcomes in the US, whereas a proposed Medicare for All plan would improve them [85]. Indeed, Medicare for All represents a significant structural intervention with potential to vastly improve care engagement among diverse MSM through facilitating standardization of the health sector. In this case, rapid assessment of patterns of care access and engagement in the wake of large-scale policy change, such as the passage of Medicare for All, would allow public health professionals to better understand the impacts of such changes, and provide timely, evidence-based recommendations to relevant policy makers in the short-term as a result. Standardized approaches to impact evaluation of structural changes in the short term would allow for broader, comprehensive work in this regard. For example, the creation of NIH funding opportunities with unique timescales designed to support in-depth evaluations on the impact of structural changes, including but not limited to changes to the landscape of health insurance, for health disparity populations. Such funding could function in a manner similar to the RADx-UP (Rapid Acceleration of Diagnostics in Underserved Populations) funding made available in response to the COVID-19 pandemic, in that it would be designed for rapid funding to support timely investigation of key questions in the wake of large-scale events or structural changes [86].
Further, evaluating the medium- and long-term impact of policy change on priority populations' health would be made substantially easier through improved collection of sex, sexual orientation, and gender identity (SSOGI) demographic data in epidemiologic health surveillance systems, including the Youth Risk Behavior Survey, Behavioral Risk Factor Surveillance System, as well as more routine inclusion in electronic health record data. The ability to accurately identify priority populations in such surveillance systems is vital to studying the wide range of health needs of marginalized communities and to understanding changes in health outcomes at the population level over time. Unfortunately, current health data captured by systems fall far short of appropriately identifying and categorizing respondents from SGM populations. There have furthermore been significant barriers in uniformly applying such data capture tools, including a lack of validated measures, as well as low willingness from policy makers and health care professionals to make changes to these systems [62]. Were such systems to include these data, ecological associations could more easily be drawn between patterns in health outcomes over time at the population level, and the implementation of structural changes. Moreover, this would simplify the process of evaluating the unintended consequences of structural change, as has been previously recommended [87].

As with structural intervention development and implementation, we recommend that all evaluation of the impact of structural interventions be conducted in a communityengaged and culturally-responsive manner. Particularly in the case of structural interventions targeting the health of vulnerable and underserved populations, it will be vital that "success" is defined not solely by academics, but by community members as well [36].

\section{Limitations}

As with any study, there were limitations to our approach that affected our work. Chief among these is that we used strict criteria for inclusion of manuscripts in this review. It is likely that, with looser criteria, we would have identified a greater number of manuscripts to include. This may include manuscripts that evaluated but did not implement structural changes or those that included but did not focus on MSM. However, the rigidity of our approach should also be considered a strength. Due to our rigid inclusion criteria, we were able to identify a notable gap in evidence-based literature testing structural interventions for HIV treatment and prevention among Black and Latinx MSM in the US. Moreover, we have accounted for this limitation by including detail on the focus of studies which were screened but not included (Fig. 2). Similarly, there is always a possibility that, despite the exhaustive nature of our search, structural interventions 
may have been missed, though this risk is likely minimal. It should also be noted that, although we created clear processes and criteria for screening and inclusion, there is a degree of subjectivity inherent in the process of selecting articles for inclusion in any systematic review. We accounted for this limitation through creating clear processes including following the evidence-based PRISMA guidance, through including multiple reviewers, and through in-depth discussion in the case of final determination. As such, we are confident that our work accurately represents the state of the literature. That said, though this may represent the current state of the published literature, it is also important to recognize that more structural interventions may be in progress that have not been formally published on yet, given the length of time it takes for the results of a structural intervention to be realized. While our search of NIH RePORTER indicates a lack of nationally-funded in-progress structural interventions, more localized efforts may be in progress. This calls for continued review of both the literature and ongoing research as new evidence on structural interventions may emerge. We also limited our search to results published in peer-reviewed academic journals, excluding grey literature. However, a number of structural interventions and evaluations take place outside of academia, especially within the context of community activism, which has propelled many of the major innovations in HIV policy. As such, further analyses, specifically of grey literature, is needed.

\section{Conclusions}

Individual-level behavioral interventions are important components of public health, particularly when it comes to infectious disease prevention. However, these smaller-scale and "downstream" approaches have less power to impact population-level prevention than interventions conducted on a population scale. Moreover, focusing solely on behavioral change in public health can obscure the impact of systems of oppression and power, which place marginalized populations at greater risk for negative health outcomes through the false perception that heath is purely a consequence of individual action and choice. Therefore, not only should behavioral interventions be designed with structural considerations in mind, but there is a need for additional interventions targeting structural factors themselves.

In reviewing the literature, we identified a key gap in the development, implementation, and evaluation of structurallevel interventions aimed at HIV prevention among Black and Latinx MSM in the US. Researchers, funders, and policy makers need to take action by prioritizing the creation, testing, and application of novel structural approaches to addressing the HIV epidemic. This work should focus on addressing structural stigma, and should center marginalized populations including MSM, transgender women, Black and Latinx people, and more. Moreover, our ability to assess the impact of structural policy changes on health would be improved by advancing existing health surveillance systems through incorporation of better demographic data capture and creation of rapid-response initiatives to collect necessary information in the wake of such policy change.

Finally, we encourage public health practitioners to consider our role in political processes. We encourage fellow public health workers and advocates of health equity to champion structural changes that stand to benefit health equity, and against those which stand to do it harm. Public health must not be partisan-we argue that it is our obligation to speak out in our professional capacity against political action which stands to harm marginalized groups through structural change, and to promote action which would ensure safety, health, and wellbeing for all.

Supplementary Information The online version contains supplementary material available at https://doi.org/10.1007/s10461-021-03167-2.

Acknowledgements No funding was used to support this research. The study design; collection, analysis, and interpretation of data; the writing of the report; and the decision to submit the article were solely the responsibility of the authors.

Author Contributions The review article was conceptualized by GP. DM, MMR, CWC, and DF conducted the literature search. GP, DM, MMR, and DF performed data analysis. All authors drafted and critically revised the work.

Funding No funding was utilized for the current study.

\section{Compliance with Ethical Standards}

Conflict of interest The authors declare that they have no conflict of interest.

Research Involving Human and/or Animal Participants The current study did not involve human participants.

Informed Consent The current study did not require informed consent.

\section{References}

1. Centers for Disease Control and Prevention. HIV Surveillance Report, 2018 (Updated). 2020. Available at: http://www.cdc.gov/ hiv/library/reports/hiv-surveillance.html. Accessed June 29, 2020.

2. Lieb S, Fallon SJ, Friedman SR, et al. Statewide estimation of racial/ethnic populations of men who have sex with men in the U.S. Public Health Rep. 2011;126(1):60-72.

3. Bronfenbrenner U. Ecological systems theory. In: Vasta R, editor. Six theories of child development: revised formulations and current issues. London: Jéssica Kingsley Publishers; 1992.

4. Levy ME, Wilton L, Phillips G, et al. Understanding structural barriers to accessing HIV testing and prevention services among 
black men who have sex with men (BMSM) in the United States. AIDS Behav. 2014;18(5):972-96.

5. Sheehan DM, Dawit R, Gbadamosi SO, et al. Sustained HIV viral suppression among men who have sex with men in the MiamiDade County Ryan White Program: the effect of demographic, psychosocial, provider and neighborhood factors. BMC Public Health. 2020;20(1):1-12.

6. Malebranche DJ, Peterson JL, Fullilove RE, Stackhouse RW. Race and sexual identity: perceptions about medical culture and healthcare among Black men who have sex with men. J Natl Med Assoc. 2004;96(1):97-107.

7. American College of Physicians. Racial and ethnic disparities in health care, updated 2010. Washington, D.C.: American College of Physicians; 2010.

8. Pager D, Shepherd H. The sociology of discrimination: racial discrimination in employment, housing, credit, and consumer markets. Annu Rev Sociol. 2008;34:181-209.

9. Li MJ, Okafor CN, Gorbach PM, Shoptaw S. Intersecting burdens: Homophobic victimization, unstable housing, and methamphetamine use in a cohort of men of color who have sex with men. Drug Alcohol Depend. 2018;192:179-85.

10. Brewer RM, Heitzeg NA. The racialization of crime and punishment: criminal justice, color-blind racism, and the political economy of the prison industrial complex. Am Behav Sci. 2008;51(5):625-44.

11. Kosciw JG, Palmer NA, Kull RM, Greytak EA. the effect of negative school climate on academic outcomes for LGBT youth and the role of in-school supports. J Sch Violence. 2013;12(1):45-63.

12. Ayala G, Bingham T, Kim J, Wheeler DP, Millett GA. Modeling the impact of social discrimination and financial hardship on the sexual risk of HIV among latino and black men who have sex with men. Am J Pub Health. 2012;102:S242-9.

13. Khan MR, McGinnis KA, Grov C, et al. Past year and prior incarceration and HIV transmission risk among HIV-positive men who have sex with men in the US. AIDS Care. 2019;31(3):349-56.

14. Sumartojo E. Structural factors in HIV prevention: concepts, examples, and implications for research. AIDS. 2000;14:S3-10.

15. Pachankis JE, Hatzenbuehler ML, Hickson F, et al. Hidden from health: structural stigma, sexual orientation concealment, and HIV across 38 countries in the European MSM Internet Survey. AIDS. 2015;29(10):1239-46.

16. Nanin J, Osubu T, Walker J, Powell B, Powell D, Parsons J. "HIV is still real": perceptions of HIV testing and HIV prevention among black men who have sex with men in New York City. Am J Mens Health. 2009;3(2):150-64.

17. National Institutes of Health. NIH RePORTER. 2020. Accessed May 292020.

18. Wohlfeiler DEJ. The limits of behavioral interventions for HIV prevention. In: Prevention is primary: strategies for community well-being. Oakland: Prevention Institute; 2007.

19. Leviton LC, Snell E, McGinnis M. Urban issues in health promotion strategies. Am J Public Health. 2000;90(6):863-6.

20. Ko JE, Jang Y, Park NS, Rhew SH, Chiriboga DA. Neighborhood effects on the self-rated health of older adults from four racial/ ethnic groups. Soc Work Public Health. 2014;29(2):89-99.

21. Dasgupta S, Kramer MR, Rosenberg ES, Sanchez TH, Reed L, Sullivan PS. The effect of commuting patterns on HIV care attendance among men who have sex with men (MSM) in Atlanta, Georgia. JMIR Public Health Surveill. 2015;1(2):e10.

22. Ransome Y, Kawachi I, Braunstein S, Nash D. Structural inequalities drive late HIV diagnosis: the role of black racial concentration, income inequality, socioeconomic deprivation, and HIV testing. Health Place. 2016;42:148-58.

23. Locher C, Messerli M, Gaab J, Gerger H. Long-term effects of psychological interventions to improve adherence to antiretroviral treatment in HIV-infected persons: a systematic review and metaanalysis. AIDS Patient Care STDS. 2019;33(3):131-44.

24. Lyles CM, Kay LS, Crepaz N, et al. Best-evidence interventions: Findings from a systematic review of HIV behavioral interventions for US populations at high risk, 2000-2004. Am J Public Health. 2007;97(1):133-43.

25. Hardeman RR, Murphy KA, Karbeah J, Kozhimannil KB. Naming Institutionalized Racism in the Public Health Literature: a systematic literature review. Public Health Rep. 2018;133(3):240-9.

26. Gupta GR, Parkhurst JO, Ogden JA, Aggleton P, Mahal A. Series HIV Prevention 4 Structural approaches to HIV prevention. Lancet. 2008;372(9640):764-75

27. Forsyth AD, Valdiserri RO. A state-level analysis of social and structural factors and HIV outcomes among men who have sex with men in the United States. AIDS Educ Prev. 2015;27(6):493-504.

28. Millett GA, Peterson JL, Flores SA, et al. Comparisons of disparities and risks of HIV infection in black and other men who have sex with men in Canada, UK, and USA: a meta-analysis. Lancet. 2012;380(9839):341-8.

29. Blankenship KM, Bray SJ, Merson MH. Structural interventions in public health. AIDS. 2000;14:S11-21.

30. Mugavero MJ, Norton WE, Saag MS. Health care system and policy factors influencing engagement in HIV medical care: piecing together the fragments of a fractured health care delivery system. Clin Infect Dis. 2011;52:S238-46.

31. Meyer IH. Minority stress and mental health in gay men. J Health Soc Behav. 1995;36(1):38-56.

32. Singer M, Bulled N, Ostrach B, Mendenhall E. Syndemics and the biosocial conception of health. Lancet. 2017;389(10072):941-50.

33. Prado G, Lightfoot $\mathrm{M}$, Brown $\mathrm{CH}$. Macro-level approaches to HIV prevention among ethnic minority youth state of the science, opportunities, and challenges. Am Psychol. 2013;68(4):286-99.

34. Zeglin RJ, Stein JP. Social determinants of health predict state incidence of HIV and AIDS: a short report. AIDS Care. 2015;27(2):255-9.

35. Adimora AA, Auerbach JD. Structural interventions for HIV prevention in the United States. J Acquir Immune Defic Syndr. 2010;55:S132-5.

36. Brown AF, Ma GX, Miranda J, et al. Structural interventions to reduce and eliminate health disparities. Am J Public Health. 2019;109(S1):S72-8.

37. Latkin CA, German D, Vlahov D, Galea S. Neighborhoods and HIV a social ecological approach to prevention and care. Am Psychol. 2013;68(4):210-24.

38. Eberhart MG, Yehia BR, Hillier A, et al. Behind the cascade: analyzing spatial patterns along the HIV care continuum. J Acquir Immune Defic Syndr. 2013;64:S42-51.

39. Viner RM, Ozer EM, Denny S, et al. Adolescent health 2 adolescence and the social determinants of health. Lancet. 2012;379(9826):1641-52.

40. Culhane DP, Gollub E, Kuhn R, Shpaner M. The co-occurrence of AIDS and homelessness: results from the integration of administrative databases for AIDS surveillance and public shelter utilisation in Philadelphia. J Epidemiol Community Health. 2001;55(7):515-20.

41. Barrington C, Acevedo R, Donastorg Y, Perez M, Kerrigan D. 'HIV and work don't go together': Employment as a social determinant of HIV outcomes among men who have sex with men and transgender women in the Dominican Republic. Glob Public Health. 2017;12(12):1506-21.

42. Jaiswal J, Griffin M, Singer SN, et al. Structural barriers to preexposure prophylaxis use among young sexual minority men: the P18 Cohort Study. Curr HIV Res. 2018;16(3):237-49.

43. Arnold T, Brinkley-Rubinstein L, Chan PA, et al. Social, structural, behavioral and clinical factors influencing retention in 
Pre-Exposure Prophylaxis (PrEP) care in Mississippi. PLoS ONE. 2017;12(2):e0172354.

44. Rebeiro PF, Howe CJ, Rogers WB, et al. The relationship between adverse neighborhood socioeconomic context and HIV continuum of care outcomes in a diverse HIV clinic cohort in the Southern United States. AIDS Care. 2018;30(11):1426-34.

45. Burke-Miller JK, Weber K, Cohn SE, et al. Neighborhood community characteristics associated with HIV disease outcomes in a cohort of urban women living with HIV. AIDS Care. 2016;28(10):1274-9.

46. Ridgway JP, Almirol EA, Schmitt J, Schuble T, Schneider JA. Travel time to clinic but not neighborhood crime rate is associated with retention in care among HIV-positive patients. AIDS Behav. 2018;22(9):3003-8.

47. Rhodes SD, Hergenrather KC, Vissman AT, et al. Boys must be men, and men must have sex with women: a qualitative CBPR study to explore sexual risk among African American, Latino, and White gay men and MSM. Am J Mens Health. 2011;5(2):140-51.

48. Santos GM, Beck J, Wilson PA, et al. Homophobia as a barrier to HIV prevention service access for young men who have sex with men. J Acquir Immune Defic Syndr. 2013;63(5):E167-71.

49. Surratt HL, Kurtz SP, Levi-Minzi MA, Chen MX. Environmental influences on HIV medication adherence: the role of neighborhood disorder. Am J Public Health. 2015;105(8):1660-6.

50. Phelan JC, Link BG, Tehranifar P. Social conditions as fundamental causes of health inequalities: theory, evidence, and policy implications. J Health Soc Behav. 2010;51:S28-40.

51. Hatzenbuehler ML. Structural stigma: research evidence and implications for psychological science. Am Psychol. 2016;71(8):742-51.

52. Brewer RA, Magnus M, Kuo I, Wang L, Liu TY, Mayer KH. The high prevalence of incarceration history among black men who have sex with men in the United States: associations and implications. Am J Public Health. 2014;104(3):448-54.

53. Meyer IH, Flores AR, Stemple L, Romero AP, Wilson BDM, Herman JL. Incarceration rates and traits of sexual minorities in the United States: National Inmate Survey, 2011-2012. Am J Public Health. 2017;107(2):267-73.

54. Brewer R, Issema R, Moore M, et al. Correlates of durable viral suppression (DVS) among criminal justice-involved (CJI) black men living with HIV in Louisiana. AIDS Behav. 2019;23(11):2980-91.

55. Friedman S, Reynolds A, Scovill S, Brassier FR, Campbell R, Ballou M. An estimate of housing discrimination against same-sex couples. Libr J. 2014;139(9):28.

56. Aidala AA, Wilson MG, Shubert V, et al. Housing status, medical care, and health outcomes among people living with HIV/AIDS: a systematic review. Am J Public Health. 2016;106(1):95.

57. Moher D, Liberati A, Tetzlaff J, Altman DG, Grp P. Preferred reporting items for systematic reviews and meta-analyses: the PRISMA statement. PLoS Med. 2009;6(7):e1000097.

58. Brewer R, Daunis C, Ebaady S, et al. Implementation of a sociostructural demonstration project to improve HIV outcomes among young black men in the deep south. J Racial Ethn Health Disparities. 2019;6(4):775-89.

59. Miller RL, Janulis PF, Reed SJ, et al. Creating youth-supportive communities: outcomes from the connect-to-protect (C2P) structural change approach to youth HIV prevention. J Youth Adolesc. 2016;45(2):301-15.

60. Arnold EA, Rebchook GM, Kegeles SM. 'Triply cursed': racism, homophobia and HIV-related stigma are barriers to regular HIV testing, treatment adherence and disclosure among young Black gay men. Cult Health Sex. 2014;16(6):710-22.

61. Carey JW, Carnes N, Schoua-Glusberg A, et al. Barriers and facilitators for clinical care engagement among HIV-positive African
American and Latino men who have sex with men. AIDS Patient Care STDS. 2018;32(5):191-201.

62. Balaji AB, An Q, Smith JC, et al. High human immunodeficiency virus incidence and prevalence and associated factors among adolescent sexual minority males-3 Cities, 2015. Clin Infect Dis. 2018;66(6):936-44.

63. Centers for Disease Control and Prevention. Diagnoses of HIV Infection in the United States and Dependent Areas, 2017. Atlanta, GA: 2018. Available at: https://www.cdc.gov/hiv/pdf/ library/reports/surveillance/cdc-hiv-surveillance-report-2017vol-29.pdf. Accessed June 29, 2020.

64. Kahana SY, Jenkins RA, Bruce D, et al. Structural determinants of antiretroviral therapy use, HIV care attendance, and viral suppression among adolescents and young adults living with HIV. PLoS ONE. 2016;11(4):e0151106.

65. Maulsby C, Millett G, Lindsey K, et al. HIV among black men who have sex with men (MSM) in the United States: a review of the literature. AIDS Behav. 2014;18(1):10-25.

66. Dang BN, Giordano TP, Kim JH. Sociocultural and structural barriers to care among undocumented latino immigrants with HIV infection. J Immigr Minor Health. 2012;14(1):124-31.

67. Garcia M, Harris AL. PrEP awareness and decision-making for Latino MSM in San Antonio, Texas. PLoS ONE. 2017;12(9):e0184014.

68. Jin H, Restar A, Biello K, et al. Burden of HIV among young transgender women: factors associated with HIV infection and HIV treatment engagement. AIDS Care. 2019;31(1):125-30.

69. Kuhns LM, Mimiaga MJ, Reisner SL, Biello K, Garofalo R. Project LifeSkills - a randomized controlled efficacy trial of a culturally tailored, empowerment-based, and group-delivered HIV prevention intervention for young transgender women: study protocol. BMC Public Health. 2017;17(1):713.

70. Reisner SL, Hughto JM, Pardee DJ, Kuhns L, Garofalo R, Mimiaga MJ. LifeSkills for men (LS4M): pilot evaluation of a gender-affirmative HIV and STI prevention intervention for young adult transgender men who have sex with men. J Urban Health. 2016;93(1):189-205.

71. Yamanis TJ, Zea MC, Montiel AKR, et al. Immigration legal services as a structural HIV intervention for latinx sexual and gender minorities. J Immigr Minor Health. 2019;21(6):1365-72.

72. Clark H, Babu AS, Wiewel EW, Opoku J, Crepaz N. Diagnosed HIV infection in transgender adults and adolescents: results from the national HIV surveillance system, 2009-2014. AIDS Behav. 2017;21(9):2774-83.

73. Barrington C, Davis DA, Villa-Torres L, Carcano J, HightowWeidman L. Intersectionalities and the HIV continuum of care among gay Latino men living with HIV in North Carolina. Ethn Health. 2019. https://doi.org/10.1080/13557858.2019.1620177.

74. Centers for Disease Control and Prevention. HIV Among Latinos. 2017. Available at: https://www.cdc.gov/hiv/group/racialethnic/ hispaniclatinos/index.html. Accessed June 29, 2020.

75. Centers for Disease Control and Prevention. HIV Among African American Gay and Bisexual Men. 2018. Available at: https ://www.cdc.gov/hiv/group/msm/bmsm.html. Accessed June 29, 2020.

76. Paz-Bailey G, Noble M, Salo K, Tregear SJ. Prevalence of HIV among US female sex workers: systematic review and meta-analysis. AIDS Behav. 2016;20(10):2318-31.

77. National Institute of Mental Health. National Institute of Mental Health Strategic Plan For Research. Bethesda: National Institute of Mental Health; 2020.

78. National Institute on Alcohol Abuse and Alcoholism. National Institute on Alcohol Abuse and Alcoholism Strategic Plan 20172021. Bethesda: National Institute on Alcohol Abuse and Alcoholism; 2017. 
79. National Institute on Minority Health and Health Disparities. NIMHD science visioning research strategies. Bethesda: National Institute on Minority Health and Health Disparities; 2019.

80. Hoppe TA, Litovitz A, Willis KA, et al. Topic choice contributes to the lower rate of NIH awards to African-American/black scientists. Sci Adv. 2019;5(10): eaaw7238.

81. Ginther DK, Schaffer WT, Schnell J, et al. Race, ethnicity, and NIH research awards. Science. 2011;333(6045):1015-9.

82. Garcia J, Parker C, Parker RG, Wilson PA, Philbin MM, Hirsch JS. "You're really gonna kick us all out?" Sustaining safe spaces for community-based HIV prevention and Control among Black Men Who Have Sex with men. PLoS ONE. 2015;10(10):e0141326.

83. Grzywacz JG, Fuqua J. The social ecology of health: leverage points and linkages. Behav Med. 2000;26(3):101-15.

84. Gonzales G, Henning-Smith C. The Affordable Care Act and Health Insurance Coverage for Lesbian, gay, and bisexual adults: analysis of the behavioral risk factor surveillance system. LGBT Health. 2017;4(1):62-7.
85. Galvani AP, Parpia AS, Foster EM, Singer BH, Fitzpatrick MC. Improving the prognosis of health care in the USA. Lancet. 2020;395(10223):524-33.

86. National Institutes of Health. RADx Underserved Populations (RADx-UP). Bethesda, Maryland: National Institutes of Health; 2020. Available at: https://www.nih.gov/research-training/medic al-research-initiatives/radx/radx-programs\#radx-up. Accessed January 11, 2021.

87. Blankenship KM, Friedman SR, Dworkin S, Mantell JE. Structural interventions: concepts, challenges and opportunities for research. J Urban Health. 2006;83(1):59-72.

Publisher's Note Springer Nature remains neutral with regard to jurisdictional claims in published maps and institutional affiliations. 